\title{
Response of GaAs to fast intense laser pulses
}

\author{
J. S. Graves and R. E. Allen \\ Department of Physics, Texas A\&M University, College Station, Texas 77843-4242
}

(Received 4 May 1998)

\begin{abstract}
Motivated by recent experiments, we have performed simulations which show in detail how the electrons and ions in GaAs respond to fast intense laser pulses (with durations of order $100 \mathrm{fs}$ and intensities of order $1-10 \mathrm{TW} / \mathrm{cm}^{2}$ ). The method of tight-binding electron-ion dynamics is used, in which an arbitrarily strong radiation field is included through a time-dependent Peierls substitution. The population of excited electrons, the atomic displacements, the atomic pair-correlation function, the band structure, and the imaginary part of the dielectric function are all calculated as functions of time, during and after application of each pulse. Above a threshold intensity, which results in promotion of about $10 \%$ of the electrons to the conduction band, the lattice is destabilized and the band gap collapses to zero. This is most clearly revealed in the dielectric function $\epsilon(\omega)$, which exhibits metallic behavior and loses its structural features after 100-200 fs.
\end{abstract}

[S0163-1829(98)01843-8]

\section{INTRODUCTION}

There are two distinct mechanisms through which an intense laser pulse can destabilize the structure of a molecule or material: On a relatively long time scale ( $\gtrsim 1 \mathrm{ps})$, the energy of excited electrons can be transferred to thermal motion of the atoms. On a shorter time scale $(\sim 100 \mathrm{fs})$, the promotion of electrons to antibonding states immediately leads to repulsive interatomic forces and the possibility of nonthermal disruption.

Consider, for example, a two-atom tight-binding model with one orbital per atom. The Hamiltonian is

$$
H(r)=\left(\begin{array}{cc}
\varepsilon_{1} & V(r) \\
V(r) & \varepsilon_{2}
\end{array}\right),
$$

so the bonding and antibonding states have energies

$$
\varepsilon_{ \pm}=\frac{1}{2}\left(\varepsilon_{1}+\varepsilon_{2}\right) \pm \frac{1}{2}\left[\left(\varepsilon_{1}-\varepsilon_{2}\right)^{2}+4 V(r)^{2}\right]^{1 / 2} .
$$

Suppose that we assume the Harrison scaling rules ${ }^{1}$

$$
V(r)=a / r^{2}, \quad u(r)=b / r^{4}
$$

where $u(r)$ is the repulsive atom-atom interaction. Since the total energy is $n_{+} \varepsilon_{+}+n_{-} \varepsilon_{-}+u(r)$, where $n_{ \pm}$represents the occupancies of the states, the force on one atom is

$$
F(r)=2\left(n_{+}-n_{-}\right)\left[1+\left(\frac{\varepsilon_{1}-\varepsilon_{2}}{2 V(r)}\right)^{2}\right]^{-1 / 2} \frac{|V(r)|}{r}+4 \frac{u(r)}{r} \text {. }
$$

In the ground state, with $n_{+}-n_{-}=-2$, an equilibrium separation can be found. But if one electron is excited to the antibonding state, making $n_{+}-n_{-}=0$, the force becomes purely repulsive and the atoms will dissociate. During the past 20 years, there has been considerable interest in the analogous problem for tetrahedral semiconductors: destabilization of the covalent bonding as electrons are excited across the band gap. ${ }^{2-20}$
Here we are concerned with GaAs, which has been the subject of several experimental studies. ${ }^{15-20}$ In particular, Mazur and co-workers ${ }^{17-20}$ have carried out detailed measurements of the dielectric function and second-order susceptibility, following 1.9-eV, 70-fs pulses which span a fluence range up to $2.5 \mathrm{~kJ} / \mathrm{m}^{2}$. Their results indicate a semiconductor-to-metal transition, and suggest that the cause is a nonthermal structural change arising directly from the excitation. In this paper we report detailed simulations of the electronic and structural response of $\mathrm{GaAs}$ to fast intense laser pulses. The method of tight-binding electron-ion dynamics is used. An arbitrarily intense radiation field is included in the Hamiltonian through a time-dependent Peierls substitution. The time-dependent Schrödinger equation is solved with an algorithm which preserves orthonormality. The atomic motion is obtained from a generalized Ehrenfest theorem. For comparison with the probe phase of a pumpprobe experiment, we calculate the dielectric function using a formula that employs the matrix elements of the tightbinding Hamiltonian, and no additional parameters. ${ }^{21}$

\section{EXCITED-STATE MOLECULAR DYNAMICS}

Before investigating the full response of electrons and ions to an intense laser pulse, let us first consider a much simpler problem: the dynamics of the atoms when some fraction of the electrons are artificially promoted to excited states. We use a standard $s p^{3} s^{*}$ tight-binding Hamiltonian ${ }^{22}$ and a nonstandard repulsive potential with the form

$$
u(r)=\left(\frac{\alpha}{r^{4}}+\frac{\beta}{r^{6}}+\frac{\gamma}{r^{8}}\right) C(r) .
$$

The total energy is then

$$
E=\sum_{k} n_{k} \varepsilon_{k}+\sum_{l>l^{\prime}} u\left(R_{l l^{\prime}}\right),
$$

where $n_{k}$ is the occupancy of the electronic state labeled by $k$ (which includes the spin index), and $R_{l l}$, is the separation 
TABLE I. Tight-binding parameters in the $\mathrm{sp}^{3} \mathrm{~s}^{*}$ model for GaAs and Si, taken from Ref. 22. The dimensionless coefficients $\eta$ are defined in Ref. 1. Here $c$ and $a$, respectively, denote the cation and anion.

\begin{tabular}{lcc}
\hline \hline & GaAs & Si \\
\hline$\varepsilon_{s_{a}}$ & -2.657 & -4.200 \\
$\varepsilon_{p_{a}}$ & 3.669 & 1.715 \\
$\varepsilon_{s *_{a}}$ & 6.739 & 6.685 \\
$\varepsilon_{s_{c}}$ & -8.343 & -4.200 \\
$\varepsilon_{p_{c}}$ & 1.041 & 1.715 \\
$\varepsilon_{s *_{c}}$ & 8.591 & 6.685 \\
$\eta_{s_{a} s_{c} \sigma}$ & -1.271 & -1.504 \\
$\eta_{s_{a} p_{c} \sigma}$ & 1.529 & 1.798 \\
$\eta_{p_{a} s_{c} \sigma}$ & -1.974 & -1.798 \\
$\eta_{p_{a} p_{c} \sigma}$ & 2.386 & 1.969 \\
$\eta_{p_{a} p_{c} \pi}$ & -0.6153 & -0.5182 \\
$\eta_{s_{a} s_{c}^{*} \sigma}$ & 0.0 & 0.0 \\
$\eta_{s_{a}^{*} s_{c} \sigma}$ & 0.0 & 0.0 \\
$\eta_{s_{a}^{*} p_{c} \sigma}$ & -1.640 & -1.687 \\
$\eta_{p_{a} s_{c}^{*} \sigma}$ & 1.652 & 1.687 \\
$\eta_{s_{a}^{*} s_{c}^{*} \sigma}$ & 0.0 & 0.0 \\
\hline \hline
\end{tabular}

of ions $l$ and $l^{\prime}$. This is simply the generalization of the expression for $E$ used in tight-binding molecular dynamics for the ground state. ${ }^{23-26}$ As usual, the second term in Eq. (2.2) represents $U_{i i}-U_{e e}$, where $U_{i i}$ is the ion-ion repulsion and $U_{e e}$ is the electron-electron repulsion [which is doubly counted in the first term of Eq. (2.2)]. For spherically symmetrical and well-separated neutral atoms, $U_{i i}-U_{e e}=0$, so $u(r)$ should fall off rapidly with distance. In Eq. (2.1), we have modified the basic Harrison scaling of Eq. (1.3) by adding two higher-order terms. We have also multiplied by a cutoff function $C(r)$, which is taken to have the form of a Fermi function:

$$
C(r)=\left[\exp \left(\left(r-r_{c}\right) / r_{w}\right)+1\right]^{-1} .
$$

The cutoff distance $r_{c}$ was chosen to be midway between $1.2 r_{1}$ and $r_{2}$, where $r_{1}=2.35 \AA$ and $r_{2}=3.84 \AA$ are, respectively, the first- and second-neighbor distance: $r_{c}$ $=\left(1.2 r_{1}+r_{2}\right) / 2$. The cutoff width $r_{w}$ was chosen to be $0.1 \AA$. With these choices, the cutoff function has little effect for bond-length changes up to $30 \%$ (so that the initial stages of destabilization will be reliably described), but falls to nearly zero at the second-neighbor distance (so that there are no unphysical distant interactions). The matrix elements of the tight-binding Hamiltonian are taken to have the Harrison scaling (1.3) and the same cutoff function,

$$
H_{\alpha \beta}^{0}\left(l l^{\prime}\right)=\bar{H}_{\alpha \beta}\left(l l^{\prime}\right)\left(r_{1} / R_{l l^{\prime}}\right)^{2} C\left(R_{l l^{\prime}}\right) / C\left(r_{1}\right),
$$

where $\alpha$ and $\beta$ represent orbitals on atoms $l$ and $l^{\prime}$. $\bar{H}_{\alpha \beta}\left(l l^{\prime}\right)$ is the Hamiltonian obtained from the parameters of Table I, using the usual Slater-Koster rules. ${ }^{1}$ The superscript 0 indicates that there is not yet an applied electromagnetic field.

The parameters $\alpha, \beta$, and $\gamma$ of Eq. (2.1) were determined by fitting the cohesive energy, lattice spacing, and bulk
TABLE II. Repulsive potential parameters for GaAs and $\mathrm{Si}$. These values are appropriate when distances are measured in $\AA$ and energies in $\mathrm{eV}$.

\begin{tabular}{lccc}
\hline \hline & $\alpha$ & $\beta$ & $\gamma$ \\
\hline $\mathrm{GaAs}$ & 263.7 & -1227.5 & 3653.1 \\
$\mathrm{Si}$ & 263.2 & -1027.0 & 2631.8 \\
\hline \hline
\end{tabular}

modulus to experiment. Details of the fitting procedure are given elsewhere. ${ }^{27}$ The resulting values for both $\mathrm{GaAs}$ and $\mathrm{Si}$ are listed in Table II.

In the simulations, an eight-atom cubic cell was used. With five orbitals per atom, the Hamiltonian matrix is then $40 \times 40$. Each atom interacts with all other atoms within the cell and their replicas outside the cell. The motion of the atoms is taken to satisfy periodic boundary conditions, so the electronic states are Bloch states corresponding to this large unit cell. In calculating the Hellmann-Feynman forces on the atoms, we used the special point $\mathbf{k}=\left(\frac{1}{4}, \frac{1}{4}, \frac{1}{4}\right)(2 \pi / a)$, together with the other points which are related to it through symmetry transformations. For the GaAs interactions, we used the parameters and cutoff function described above. For Ga-Ga and As-As interactions (which are irrelevant in the initial stages of destabilization), we used the same parameters, but replaced the Fermi function cutoff (2.3) by a thetafunction cutoff $\theta\left(r_{1}-R_{l l^{\prime}}\right)$. The velocity Verlet algorithm was used, ${ }^{28,29}$ with a time step of $0.05 \mathrm{fs}$. Energy is then typically conserved to about one part in $10^{6}$ for low excitation of the electrons, or one part in $10^{4}$ at high excitations that cause more violent atomic motion. Expression (2.2) leads to the usual Hellmann-Feynman theorem of tightbinding molecular dynamics, ${ }^{23,24}$

$$
M \ddot{X}=-\sum_{k} n_{k} \boldsymbol{\Psi}_{k}^{\dagger} \cdot \frac{\partial \mathbf{H}^{0}}{\partial X} \cdot \boldsymbol{\Psi}_{k}-\frac{\partial U}{\partial X},
$$

where $X$ and $M$ are any ion coordinate and mass,

$$
U=\sum_{l>l^{\prime}} u\left(R_{l l^{\prime}}\right)
$$

and $\mathbf{H}^{0}$ is the Hamiltonian matrix of (2.4).

Figure 1 summarizes the results when the atoms are given an initial kinetic energy corresponding to $300 \mathrm{~K}$, but some fraction of the electrons are artificially promoted from the top of the valence band to the bottom of the conduction band. If $\frac{4}{32}$, or $12.5 \%$, of the electrons are promoted, the atoms are observed to move far from their equilibrium positions in the original tetrahedral structure, so the lattice has definitely been destabilized.

\section{ELECTRON-ION DYNAMICS}

Let us now turn to full simulations of the coupled dynamics of electrons and ions in a material which is subjected to an intense laser pulse. The vector potential is taken to have the time dependence 


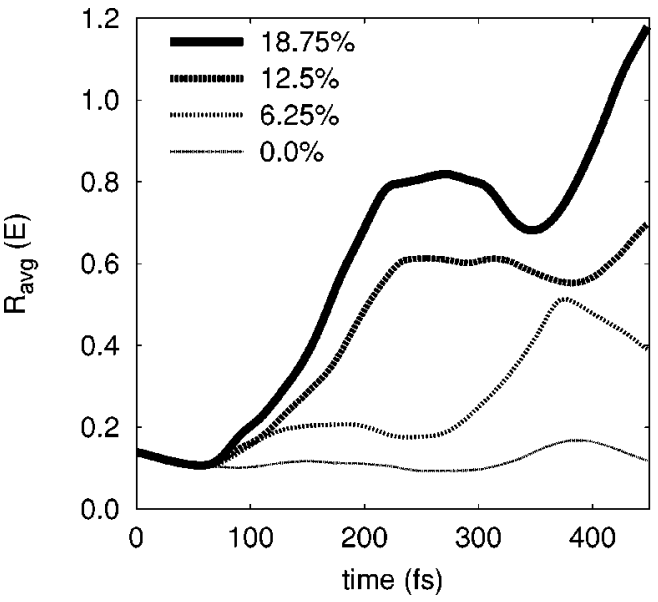

FIG. 1. Average distance moved for GaAs atoms, shown as a function of time for varying excitation levels.

$$
\mathbf{A}(t)=A_{0} \cos \left(\frac{\pi\left(t-t_{0} / 2\right)}{t_{0}}\right) \cos (\omega t), \quad 0 \leqslant t \leqslant t_{0} .
$$

This form (i) closely resembles a Gaussian, ${ }^{27}$ (ii) clips the pulse to zero at beginning and end, (iii) gives zero slope for $A(t)^{2}$ at beginning and end, and (iv) gives a full width at half maximum (FWHM) duration for $A(t)^{2}$ of exactly half the total pulse time $t_{0}$.

The usual Hellmann-Feynman theorem of Eq. (2.5) is no longer valid when the electronic states $\boldsymbol{\Psi}_{j}$ are no longer eigenstates of the Hamiltonian. However, Eq. (2.5) can be replaced by a generalized Ehrenfest theorem ${ }^{30,31}$

$$
M \ddot{X}=-\sum_{j} \boldsymbol{\Psi}_{j}^{\dagger} \cdot \frac{\partial \mathbf{H}}{\partial X} \cdot \boldsymbol{\Psi}_{j}-\frac{\partial U}{\partial X} .
$$

This equation for the ion dynamics is coupled to the timedependent Schrödinger equation for the electron dynamics, ${ }^{30,31}$

$$
i \hbar \partial \mathbf{\Psi}_{j} / \partial t=\mathbf{H}(t) \cdot \mathbf{\Psi}_{j} .
$$

The electrons are in turn coupled to the radiation field through a time-dependent Peierls substitution ${ }^{21,31}$

$$
H_{\alpha \beta}\left(l l^{\prime}\right)=H_{\alpha \beta}^{0}\left(l l^{\prime}\right) \exp \left(\frac{i e}{\hbar c} \mathbf{A} \cdot\left(\mathbf{R}_{l}-\mathbf{R}_{l^{\prime}}\right)\right),
$$

where $\mathbf{R}_{l}$ is the position of the $l$ th ion. The direct force of the electromagnetic field on the ions is omitted, since this force oscillates on a 1-fs time scale, two orders of magnitude shorter than the response time of the ions.

The second-order equation (3.2) was solved with the velocity Verlet algorithm, which preserves the phase space. On the other hand, if one tries to solve the first-order equation (3.3) with a naive algorithm, the orthonormality relations

$$
\boldsymbol{\Psi}_{i}(t) \cdot \boldsymbol{\Psi}_{j}(t)=\delta_{i j}
$$

are not preserved. Instead, we write this time evolution equation in the form

$$
\exp \left(\frac{i \mathbf{H} \Delta t}{2 \hbar}\right) \cdot \boldsymbol{\Psi}_{j}(t+\Delta t)=\exp \left(\frac{-i \mathbf{H} \Delta t}{2 \hbar}\right) \cdot \boldsymbol{\Psi}_{j}(t)
$$

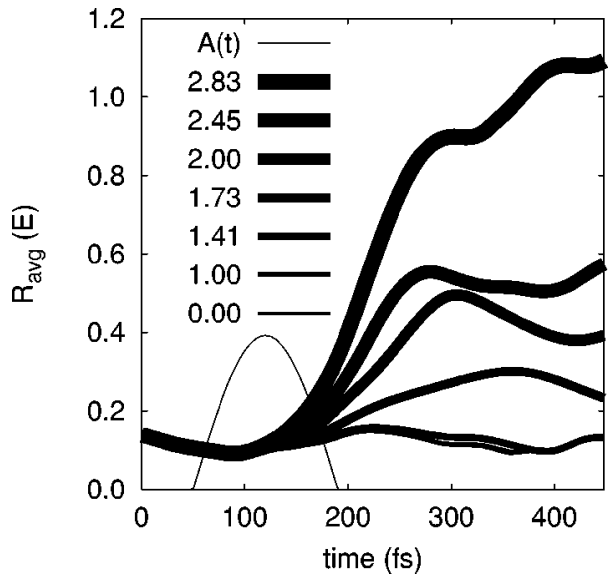

FIG. 2. Average distance moved by GaAs atoms, during and following a laser pulse. The amplitude $A_{0}$ of the vector potential is given in $\mathrm{G} \mathrm{cm}$.

and then retain the first two terms in each exponential. This yields the Cayley algorithm

$$
\boldsymbol{\Psi}_{j}(t+\Delta t)=(\mathbf{1}+i \mathbf{H} \Delta t / 2 \hbar)^{-1} \times(\mathbf{1}-i \mathbf{H} \Delta t / 2 \hbar) \cdot \boldsymbol{\Psi}_{j}(t) .
$$

With a time step $\Delta t=0.05 \mathrm{fs}$, orthonormality is then preserved to about three parts in $10^{6}$ during a simulation of $500 \mathrm{fs}$.

It is clear that the present method involves a number of approximations: (1) Since it employs a one-electron picture, electron-electron interactions are omitted. As a result, there are no excitonic corrections or carrier-carrier scattering. Although these effects are of central importance in other experiments, we feel that they are not relevant in the initial stages of the nonthermal phase transitions studied here and observed in Refs. 16-20. (2) Since the atomic motion is treated classically, there are no phonon cascades following excitation. Quantized emission of phonons, like carriercarrier interactions (and the weaker effect of spontaneous emission of photons), will be important on a longer time scale, but again is not a large effect in the early stages of electronic excitation and lattice destabilization which are considered here. (3) Band-gap renormalization due to carrier screening will also produce reduction of the band gap when a semiconductor is subjected to an intense laser pulse. The present calculations are therefore complementary to previous calculations which treat only carrier screening, ${ }^{11}$ and which omit the structural changes and other effects that are included in the present treatment. The present calculations imply that the dominant effect in the experiments of Refs. 16-20 is lattice destabilization due to excitation of electrons from bonding to antibonding states, and that this effect is sufficient to explain the observations, so that there is no need to invoke secondary mechanisms like band-gap renormalization.

\section{RESULTS}

Figure 2 shows the atomic motion that results when laser pulses of various intensities are applied to GaAs initially in thermal equilibrium at $300 \mathrm{~K}$ (after an equilibration period of $2000 \mathrm{fs}$ ). In each case the FWHM pulse duration was $70 \mathrm{fs}$, 


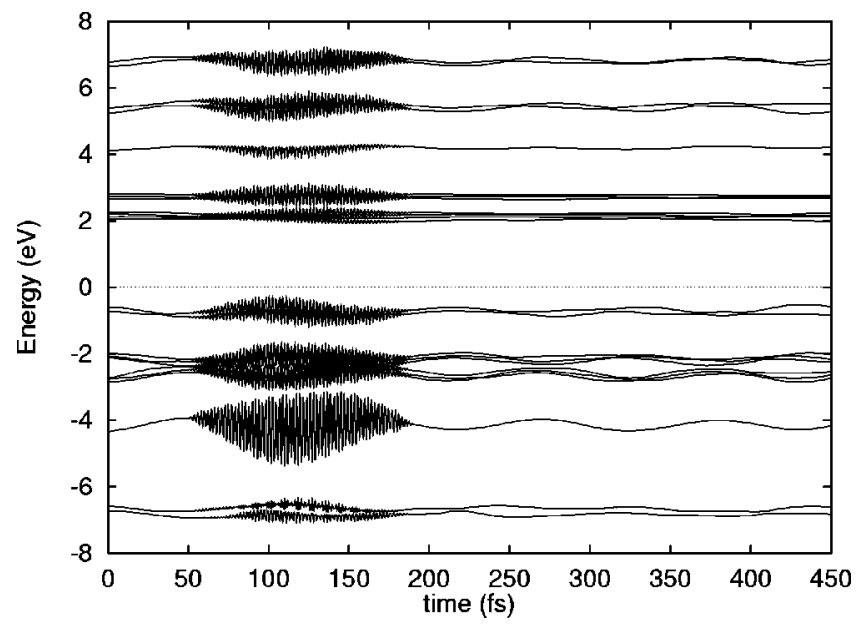

FIG. 3. Electronic energy eigenvalues at the $(2 \pi / a)\left(\frac{1}{4}, \frac{1}{4}, \frac{1}{4}\right)$ point as a function of time, with $A_{0}=1.00 \mathrm{G} \mathrm{cm}$.

with $\hbar \omega=1.95 \mathrm{eV}$, and a polarization in the $(1.7,1.0,0) \mathrm{di}-$ rection, referenced to the cube edges.

As shown elsewhere, ${ }^{27}$ an amplitude $A_{0}=1.00 \mathrm{G} \mathrm{cm}$ corresponds to a fluence of $0.815 \mathrm{~kJ} / \mathrm{m}^{2}$. The threshold for permanent structural change is about $2.00 \mathrm{G} \mathrm{cm}$, or $3.26 \mathrm{~kJ} / \mathrm{m}^{2}$. This is about three times as large as the experimental threshold. ${ }^{16-20}$ Since the present theory yields a dielectric function which is roughly half that observed experimentally, ${ }^{21}$ one expects the nonlinear response to also be underestimated, so this level of agreement is quite satisfactory.

Although $\boldsymbol{\Psi}_{j}(t)$ is the physical state for the $j$ th electron, one can also define eigenvectors $\boldsymbol{\Phi}_{n}(\mathbf{k})$ of the timedependent Hamiltonian:

$$
\mathbf{H} \cdot \boldsymbol{\Phi}_{n}(\mathbf{k})=\varepsilon_{n}(\mathbf{k}) \boldsymbol{\Phi}_{n}(\mathbf{k}) .
$$

The eigenvalues $\varepsilon_{n}(\mathbf{k})$ at the special point $\mathbf{k}$ $=\left(\frac{1}{4}, \frac{1}{4}, \frac{1}{4}\right)(2 \pi / a)$ are plotted as functions of time in Figs. 3 and 4 , for two different intensities. Notice that the band gap at this point [which is larger than the fundamental band gap

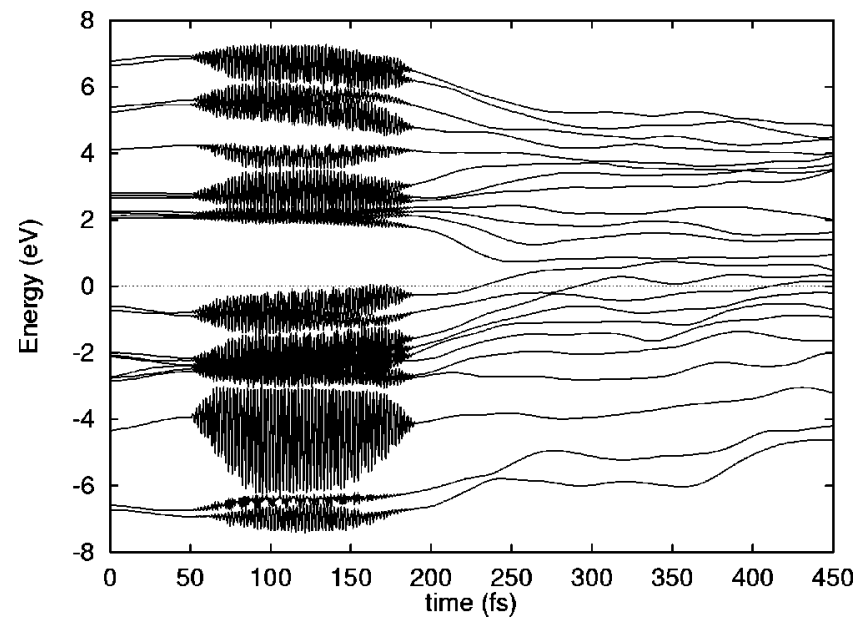

FIG. 4. Electronic energy eigenvalues at the $(2 \pi / a)\left(\frac{1}{4}, \frac{1}{4}, \frac{1}{4}\right)$ point as a function of time, with $A_{0}=2.83 \mathrm{G} \mathrm{cm}$.

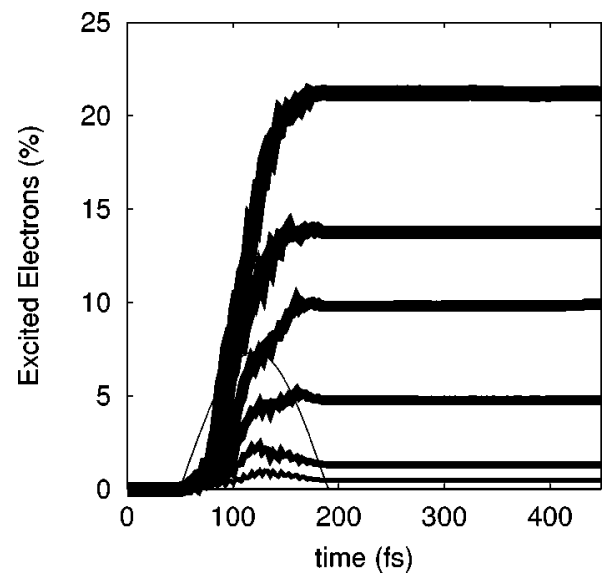

FIG. 5. The percentage of valence electrons promoted to excited states is shown as a function of time for varying pulse intensities. The pulse is represented by a solid curve. The amplitudes $A_{0}$ are given in Fig. 2.

at $(0,0,0)]$ exhibits only thermal oscillations for $A_{0}$ $=1.00 \mathrm{Gcm}$, but has completely closed up for $A_{0}$ $=2.83 \mathrm{G} \mathrm{cm}$ because of the large atomic displacements associated with lattice destabilization. The rapid oscillations during application of the pulse are due to the Peierls factor in Eq. (3.4).

The occupancy of the $k$ th state is given by

$$
n_{k}=\sum_{j}\left|\boldsymbol{\Psi}_{j}^{\dagger} \cdot \boldsymbol{\Phi}_{k}\right|^{2}
$$

where $k \leftrightarrow \mathbf{k}, n$. The total occupancy of all the conduction bands (again at the special point) is plotted as a function of time in Fig. 5, where it is expressed as a percentage of the total number of valence electrons. Since our model does not include spontaneous emission or carrier interactions, $n_{k}$ remains constant after the pulse is turned off. Notice that the threshold for permanent structural change corresponds to excitation of about $10 \%$ of the valence electrons.

In Figs. 6 and 7, the pair-correlation function is plotted as a function of time. The structural order remains intact for $A_{0}=1.00 \mathrm{Gcm}$, but is lost after about 200 fs for $A_{0}$ $=2.83 \mathrm{G} \mathrm{cm}$, confirming that this higher-field strength leads to a permanent structural change.

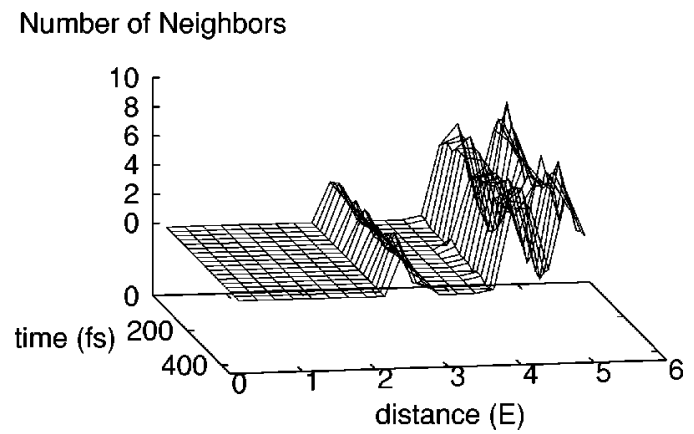

FIG. 6. Time evolution of the pair correlation function for a field strength of $A_{0}=1.00 \mathrm{G} \mathrm{cm}$. 


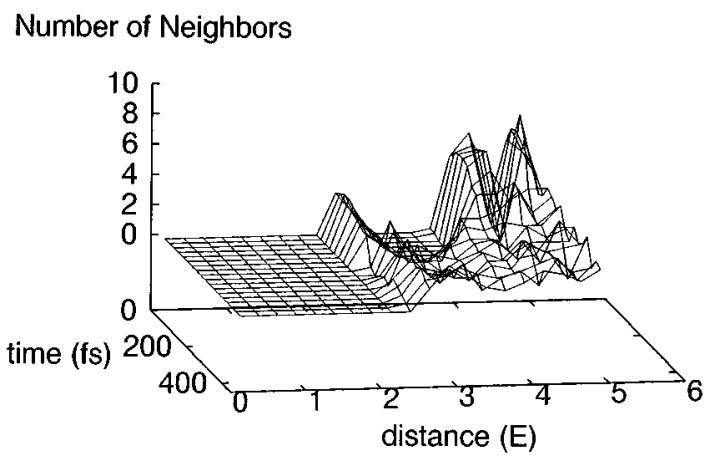

FIG. 7. Time evolution of the pair correlation function for $A_{0}$ $=2.83 \mathrm{G} \mathrm{cm}$.

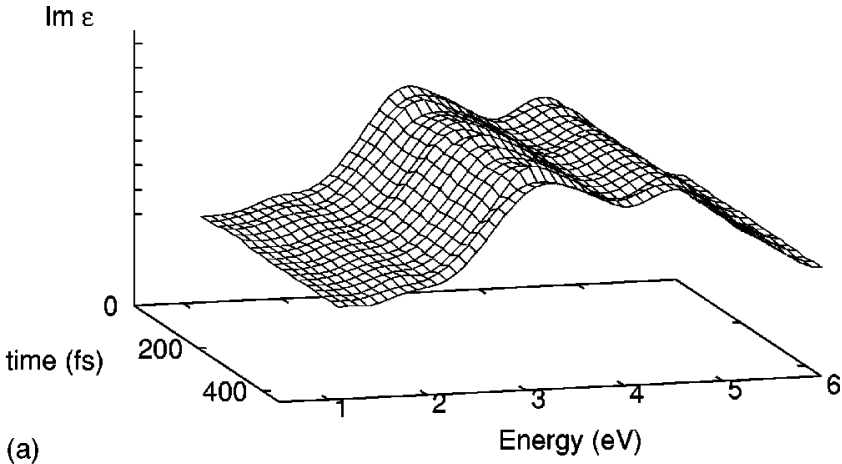

(a)

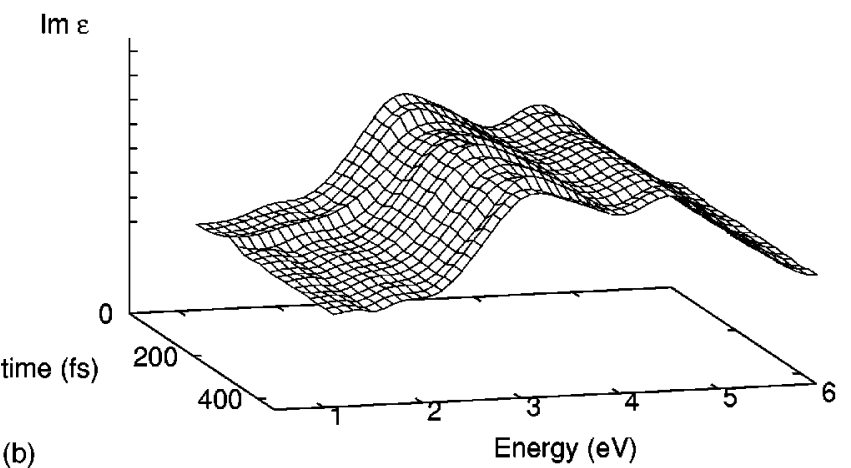

(b)

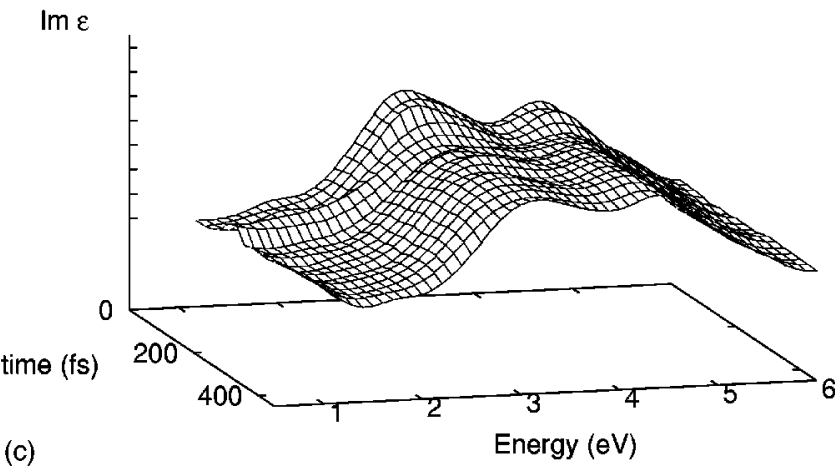

FIG. 8. Time-dependent dielectric function for $0.5 \mathrm{eV} \leqslant \hbar \omega$ $\leqslant 6.0 \mathrm{eV}$ and for three subthreshold intensities: $A_{0}=1.00,1.41$, and $1.73 \mathrm{G} \mathrm{cm}$. Im $\boldsymbol{\epsilon}(\omega)$ is shown for a time interval of $450 \mathrm{fs}$, with the pulse applied between 50 and $190 \mathrm{fs}$.
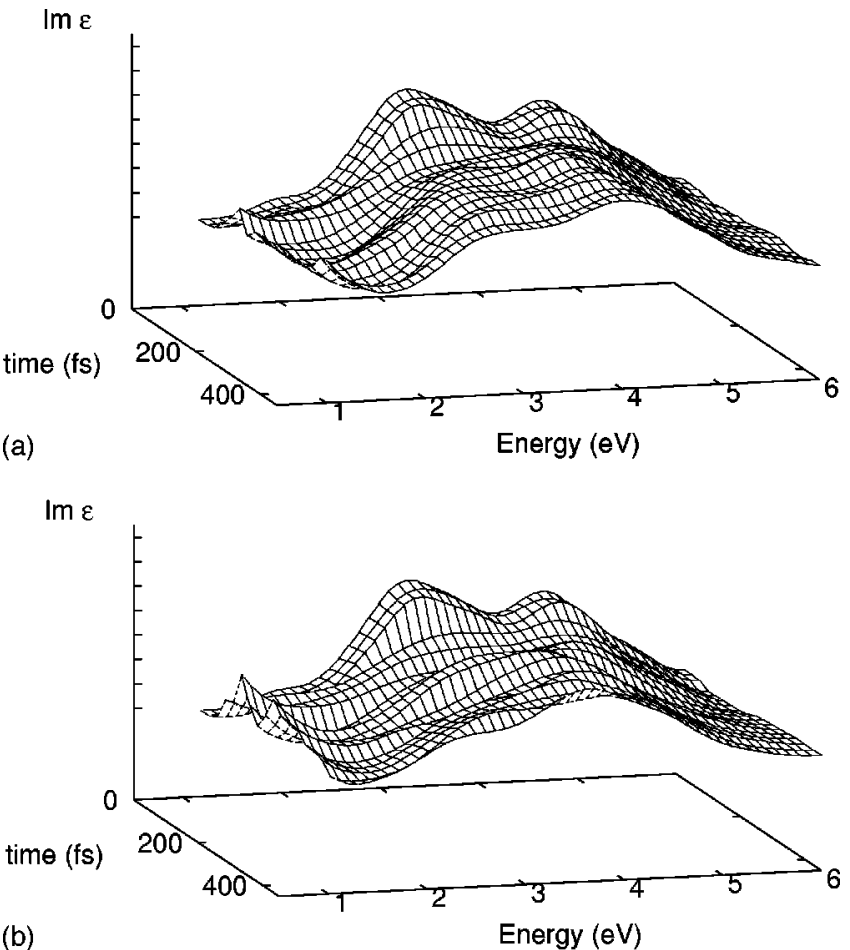

(b)

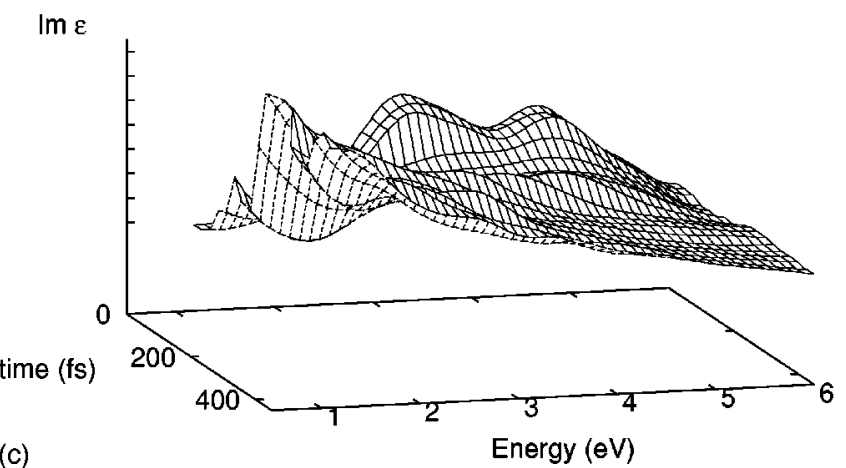

FIG. 9. Im $\epsilon(\omega)$ for three above-threshold intensities: $A_{0}$ $=2.00,2.45$, and $2.83 \mathrm{G} \mathrm{cm}$.

The most direct comparison with experiment is provided by the imaginary part of the dielectric function, ${ }^{16-20}$ which can be calculated from the formula ${ }^{21}$

$$
\begin{aligned}
\operatorname{Im} \varepsilon(\omega) \propto & \frac{1}{\omega^{2}} \sum_{n, m, \mathbf{k}}\left[f_{n}(\mathbf{k})-f_{m}(\mathbf{k})\right] \\
& \times \mathbf{p}_{n m}(\mathbf{k}) \cdot \mathbf{p}_{m n}(\mathbf{k}) \delta\left[\omega-\omega_{m n}(\mathbf{k})\right],
\end{aligned}
$$

where $\omega_{m n}(\mathbf{k})=\left[\varepsilon_{m}(\mathbf{k})-\varepsilon_{n}(\mathbf{k})\right] / \hbar, f_{n}(\mathbf{k})$ is the same as the occupancy of Eq. (4.2) (with $k \leftrightarrow \mathbf{k}, n$ ), and $\mathbf{p}_{n m}(\mathbf{k})$ is defined in Ref. 21. It is legitimate to define a time-dependent dielectric function in the present context, because the time scale for changes in the electronic structure $(\sim 200 \mathrm{fs})$ is much longer than the time scale for oscillations of the field $(\sim 2 \mathrm{fs})$. In the summation of Eq. (4.3), the following $512 \mathbf{k}$ points were included:

$$
\mathbf{k}_{n_{1}, n_{2}, n_{3}}=\frac{1}{16}\left(n_{1}, n_{2}, n_{3}\right),
$$

with 


$$
n_{1}, n_{2}, n_{3}= \pm 1, \pm 3, \pm 5, \pm 7 \text {. }
$$

Also, the $\delta$ function was approximated by a Gaussian,

$$
\delta(\omega) \approx \frac{1}{\sqrt{\pi}} \frac{e^{-(\hbar \omega / \delta \varepsilon)^{2}}}{\delta \varepsilon / \hbar},
$$

with $\delta \varepsilon=0.3 \mathrm{eV}$.

The panels of Figs. 8 and 9 show the imaginary part of the dielectric function for $0.5 \mathrm{eV} \leqslant \hbar \omega \leqslant 6.0 \mathrm{eV}$ and for six different intensities, ranging from $A_{0}=1.00$ to $A_{0}$ $=2.83 \mathrm{G} \mathrm{cm}$. (The corresponding fluences range from 0.8 to $6.5 \mathrm{~kJ} / \mathrm{m}^{2}$.) At low intensities there is no absorption for $\hbar \omega$ less than the band gap of $1.5 \mathrm{eV}$ [i.e., $\operatorname{Im} \varepsilon(\omega)$ is zero in this range] and the structural features in $\operatorname{Im} \varepsilon(\omega)$ persist at all time. At high intensities, one can observe metallic behavior (with subband-gap absorption) and the structural features are washed out. These conclusions are consistent with the measurements. ${ }^{16-20}$ Once again, the threshold in the simulations is at about $A_{0}=2.00 \mathrm{G} \mathrm{cm}$, corresponding to excitation of about $10 \%$ of the valence electrons.

\section{CONCLUSION}

We have performed simulations of the electronic and structural response of GaAs to ultraintense and ultrashort pulses. The fluence was taken to range from zero up to $6.5 \mathrm{~kJ} / \mathrm{m}^{2}$, with a FWHM pulse duration of $70 \mathrm{fs}$ and $\hbar \omega$ $=1.95 \mathrm{eV}$. We employed a method for treating nonadiabatic processes: tight-binding electron-ion dynamics. ${ }^{31}$ An arbitrarily strong radiation field is included in the electronic Hamiltonian through a time-dependent Peierls substitution. The time-dependent Schrödinger equation is solved with an algorithm which preserves orthonormality, and the atomic motion is obtained from a generalized Ehrenfest theorem.

These simulations provide a detailed microscopic understanding of the behavior of electron and ions. As can be seen in Fig. 5, the density of excited electrons increases with the intensity (or fluence) of the pulse; for $A_{0}=2.0 \mathrm{G} \mathrm{cm}$, about $10 \%$ of the valence electrons are excited into the conduction bands. Figure 2 shows that this represents the approximate threshold for atoms to perform large excursions from their initial positions, corresponding to permanent structural change. A threshold at about $10 \%$ excitation is also consistent with the excited-state molecular-dynamics simulation of Fig. 1. The fact that there is a structural transformation is confirmed by calculation of the pair correlation fuction, as in Fig. 7.

Accompanying the structural transformation at higher intensities, there is a collapse of the band-gap, which can be clearly seen in Fig. 4 at the single $\mathbf{k}$ point $\left(\frac{1}{4}, \frac{1}{4}, \frac{1}{4}\right)(2 \pi / a)$. A better measure of the onset of metallic behavior is provided by the dielectric function $\varepsilon(\omega)$. As can be seen in Fig. 9, $\operatorname{Im} \varepsilon(\omega)$ becomes nonzero within the band gap (i.e., for $\hbar \omega<1.5 \mathrm{eV}$ ) when $A_{0}$ exceeds the threshold value of 2.0 $\mathrm{Gcm}$. The simulations presented here are fully consistent with the experiments, ${ }^{16-20}$ and they provide a detailed microscopic picture for the coupled response of electrons and ions when a fast intense laser pulse is applied to a semiconductor.

\section{ACKNOWLEDGMENTS}

We thank the authors of Refs. 19 and 20 for their suggestions and for preprints of their most recent papers. This work was supported by the Robert A. Welch Foundation.
${ }^{1}$ W. A. Harrison, Electronic Structure and the Properties of Solids (Freeman, San Francisco, 1980).

${ }^{2}$ E. Mazur, in Spectroscopy and Dynamics of Collective Excitations in Solids, edited by B. D. Bartolo (Plenum, New York, 1996).

${ }^{3}$ D. von der Linde, in Ultrashort Laser Pulses and Applications, edited by W. Kaiser (Springer-Verlag, Berlin, 1988).

${ }^{4}$ J. A. Van Vechten, in Semiconductors Probed by Ultrafast Laser Spectroscopy, edited by R. R. Alfano (Academic, London, 1984).

${ }^{5}$ J. A. Van Vechten, R. Tsu, and F. W. Saris, Phys. Lett. 74A, 422 (1979).

${ }^{6}$ J. Bok, Phys. Lett. 84A, 448 (1981).

${ }^{7}$ M. Combescot and J. Bok, Phys. Rev. Lett. 48, 1413 (1982).

${ }^{8}$ H. M. Van Driel, in Semiconductors Probed by Ultrafast Laser Spectroscopy (Ref. 4).

${ }^{9}$ H. M. van Driel, Phys. Rev. B 35, 8166 (1987).

${ }^{10}$ S. Das Sarma and J. R. Senna, Phys. Rev. B 49, 2443 (1994).

${ }^{11}$ D. H. Kim, H. Ehrenreich, and E. Runge, Solid State Commun. 89, 119 (1994).

${ }^{12}$ P. Stampfli and K. H. Bennemann, Phys. Rev. B 42, 7163 (1990).

${ }^{13}$ P. L. Silvestrelli, A. Alavi, M. Parrinello, and D. Frenkel, Phys. Rev. B 77, 3149 (1996).

${ }^{14}$ C. V. Shank, R. Yen, and C. Hirlimann, Phys. Rev. Lett. 50, 454 (1983).
${ }^{15}$ S. V. Govorkov, Th. Schröder, I. L. Shumay, and P. Heist, Phys. Rev. B 46, 6864 (1992).

${ }^{16}$ K. Sokolowski-Tinten, J. Bialkowski, and D. von der Linde, Phys. Rev. B 51, 14186 (1995).

${ }^{17}$ Y. Siegal, E. N. Glezer, and E. Mazur, Phys. Rev. B 49, 16403 (1994).

${ }^{18}$ Y. Siegal, E. N. Glezer, L. Huang, and E. Mazur, Annu. Rev. Mater. Sci. 25, 223 (1995).

${ }^{19}$ E. N. Glezer, Y. Siegal, L. Huang, and E. Mazur, Phys. Rev. B 51, 6959 (1995); 51, 9589 (1995).

${ }^{20}$ L. Huang, J. P. Callan, E. N. Glezer, and E. Mazur, Phys. Rev. Lett. 80, 185 (1998).

${ }^{21}$ M. Graf and P. Vogl, Phys. Rev. B 51, 4940 (1995).

${ }^{22}$ P. Vogl, H. P. Hjalmarson, and J. D. Dow, J. Phys. Chem. Solids 44, 365 (1983)

${ }^{23}$ M. Menon and R. E. Allen, Phys. Rev. B 33, 7099 (1986).

${ }^{24}$ O. F. Sankey and R. E. Allen, Phys. Rev. B 33, 7164 (1986).

${ }^{25}$ J. Gryko and R. E. Allen, in High Performance Computing and Its Application in the Physical Sciences, edited by D. A. Brown et al. (World Scientific, Singapore, 1994).

${ }^{26}$ M. Sawtarie, M. Menon, and K. R. Subbaswamy, Phys. Rev. B 49, 7739 (1994), and references therein.

${ }^{27}$ J. S. Graves, Ph.D. thesis, Texas A\&M University, 1997.

${ }^{28} \mathrm{H}$. Gould and J. Tobochnik, An Introduction to Computer Simu- 
lation Methods (Addison-Wesley, New York, 1988).

${ }^{29}$ D. W. Heerman and A. N. Burkitt, Parallel Algorithms in Compuational Science (Springer-Verlag, New York, 1991).

${ }^{30}$ R. E. Allen, Phys. Rev. B 50, 18629 (1994).
${ }^{31}$ J. Graves and R. Allen, in Tight-Binding Approach to Computational Materials Science, edited by P.E.A. Turchi, A. Gonis, and L. Colombo (Materials Research Society, Warrendale, PA, 1998). 